\title{
European Stroke Organisation (ESO) guidelines on blood pressure management in acute ischaemic stroke and intracerebral haemorrhage
}

European Stroke Journal 202I, Vol. 6(2) II

(C) European Stroke Organisation 2021 (c) (i)

Article reuse guidelines: sagepub.com/journals-permissions DOI: 10.1 I77/239698732II026998 journals.sagepub.com/home/eso (SAGE

\author{
Else Charlotte Sandset ${ }^{1,2}$ (D), Craig S Anderson ${ }^{3,4}$ D, \\ Philip M Bath ${ }^{5}$, Hanne Christensen ${ }^{6}$, Urs Fischer ${ }^{7}$, \\ Dariusz Gasecki ${ }^{8}$, Avtar Lal ${ }^{9}$, Lisa S Manning ${ }^{10}$, \\ Simona Sacco ${ }^{\prime \prime}$ (D), Thorsten Steiner ${ }^{12,13}$ (D) and \\ Georgios Tsivgoulis ${ }^{14,15}$
}

\begin{abstract}
The optimal blood pressure (BP) management in acute ischaemic stroke (AIS) and acute intracerebral haemorrhage (ICH) remains controversial. These European Stroke Organisation (ESO) guidelines provide evidence-based recommendations to assist physicians in their clinical decisions regarding BP management in acute stroke. The guidelines were developed according to the ESO standard operating procedure and Grading of Recommendations, Assessment, Development, and Evaluation (GRADE) methodology. The working group identified relevant clinical questions, performed systematic reviews and meta-analyses of the literature, assessed the quality of the available evidence, and made specific recommendations. Expert consensus statements were provided where insufficient evidence was available to provide recommendations based on the GRADE approach. Despite several large randomised-controlled clinical trials, quality of evidence is generally low due to inconsistent results of the effect of blood pressure lowering in AIS. We recommend early and modest blood pressure control (avoiding blood pressure levels $>180 / 105 \mathrm{~mm} \mathrm{Hg}$ ) in AIS patients undergoing reperfusion therapies. There is more high-quality randomised evidence for BP lowering in acute ICH, where intensive blood pressure lowering is recommended rapidly after hospital presentation with the intent to improve recovery by reducing haematoma expansion. These guidelines provide further recommendations on blood pressure thresholds and for specific patient subgroups. There is ongoing uncertainty regarding the most appropriate blood pressure management in AIS and ICH. Future randomised-controlled clinical trials are needed to inform decision making on thresholds, timing and strategy of blood pressure lowering in different acute stroke patient subgroups.
\end{abstract}

\section{Keywords}

ischaemic stroke, intracerebral haemorrhage, blood pressure, hypertension, guidelines, recommendations, antihypertensive, blood pressure lowering

Date received: 5 March 202I; accepted: 5 April 202I

\footnotetext{
'Stroke Unit, Department of Neurology, Oslo University Hospital, Oslo, Norway

${ }^{2}$ The Norwegian Air Ambulance Foundation, Oslo, Norway

${ }^{3}$ The George Institute for Global Health, Faculty of Medicine, University of New South Wales, Sydney, NSW, Australia

${ }^{4}$ The George Institute China at Peking University Health Science Center, Beijing, PR China

${ }^{5}$ Stroke Trials Unit, Division of Clinical Neuroscience, University of Nottingham, Nottingham NG7 2UH, United Kingdom

${ }^{6}$ Department of Neurology, Bispebjerg Hospital \& University of

Copenhagen, Copenhagen, Denmark

${ }^{7}$ Department of Neurology, Inselspital, Bern University Hospital, University of Bern, Bern, Switzerland

${ }^{8}$ Department of Adult Neurology, Medical University of Gdańsk, Gdańsk, Poland
}

\footnotetext{
${ }^{9}$ Methodologist, European Stroke Organisation, Basel, Switzerland

${ }^{10}$ Department of Stroke Medicine, University Hospitals of Leicester NHS Trust, Leicester, United Kingdom

"Department of Biotechnological and Applied Clinical Sciences,

University of L'Aquila, Italy

${ }^{12}$ Department of Neurology, Frankfurt Hoechst Hospital, Frankfurt, Germany

${ }^{13}$ Department of Neurology, Heidelberg University Hospital, Heidelberg, Germany

${ }^{14}$ Second Department of Neurology, Attikon University Hospital, School of Medicine, National and Kapodistrian University of Athens, Athens, Greece

${ }^{15}$ Department of Neurology, University of Tennessee Health Science Center, Memphis, Tennessee, USA
}

Corresponding author:

Else Charlotte Sandset, Stroke Unit, Department of Neurology, Oslo University Hospital, Ullevål 4950, Postboks Nydalen, Oslo 0424, Norway. Email: Else@sandset.net 